Rev. Int. Contam. Ambie. 33 (2) 221-235, 2017

DOI: 10.20937/RICA.2017.33.02.04

\title{
HIDROQUÍMICA Y CONTAMINANTES EMERGENTES EN AGUAS RESIDUALES URBANO INDUSTRIALES DE MORELIA, MICHOACÁN, MÉXICO
}

\author{
Víctor Hugo ROBLEDO ZACARÍAS ${ }^{1}$, Martha Alicia VELÁZQUEZ MACHUCA ${ }^{1 *}$, \\ José Luis MONTAÑEZ SOTO ${ }^{1}$, José Luis PIMENTEL EQUIHUA ${ }^{2}$, Alba Adriana VALLEJO CARDONA ${ }^{3}$, \\ María Dolores LÓPEZ CALVILLO ${ }^{4}$ y José VENEGAS GONZÁLEZ ${ }^{1}$
}

${ }^{1}$ Centro Interdisciplinario de Investigación para el Desarrollo Integral Regional, Unidad Michoacán, Instituto Politécnico Nacional. Justo Sierra 28, Colonia Centro, Jiquilpan, Michoacán, México, C. P. 59510

${ }^{2}$ Centro de Estudios del Desarrollo Rural, Colegio de Postgraduados. Kilómetro 36.5, Texcoco 5, México Texcoco, Montecillo, México, C. P. 56230

${ }^{3}$ Centro de Nanociencias y Micro y Nanotecnología, Instituto Politécnico Nacional. Luis Enrique Erro, Colonia Nueva Industrial Vallejo, Ciudad de México, México, C. P. 07738

${ }^{4}$ Facultad de Químico Farmacobiología, Universidad Michoacana de San Nicolás de Hidalgo. Avenida Lázaro Cárdenas 2707, Colonia Chapultepec Norte, Morelia, Michoacán, México, C. P. 58240

*Autor para correspondencia: malivelazquez@hotmail.com

(Recibido noviembre 2015; aceptado agosto 2016)

Palabras clave: productos farmacéuticos, aguas de riego, metales pesados, reúso agrícola

\section{RESUMEN}

En México, el $54 \%$ de las aguas residuales no reciben tratamiento y son desalojadas hacia los cuerpos de agua, suelos y canales de riego lo que genera un fuerte problema de contaminación y un alto riesgo para la salud humana y la biota acuática. En las últimas décadas se han detectado los denominados contaminantes emergentes en aguas superficiales con presencia de aguas residuales crudas y tratadas. Estos compuestos y sus metabolitos reactivos causan severos efectos toxicológicos en los organismos acuáticos o microorganismos del suelo aún en bajas concentraciones. El objetivo del trabajo fue determinar las propiedades físicas y químicas: $\mathrm{pH}$, conductividad eléctrica (CE), sólidos disueltos totales (SDT), $\mathrm{Ca}^{2+}, \mathrm{Mg}^{2+}, \mathrm{Na}^{+}$, boro (B), fósforo total (P), metales traza $(\mathrm{Cu}, \mathrm{Zn}, \mathrm{Fe}, \mathrm{Mn})$ e identificar y cuantificar los contaminantes emergentes (farmacéuticos y drogas de abuso) en los influentes y efluentes de la Planta de Tratamiento de Aguas Residuales (PTAR) de Morelia, utilizando metodologías estandarizadas, espectrofotometría de infrarrojo (FT-IR) y espectrometría de masas (ESI-MS-TOF). Los valores de pH, CE, SDT, relación de adsorción de sodio (RAS), B, Py metales traza se encontraron dentro de los límites marcados por la norma mexicana NOM-001-ECOL-1996 y directrices internacionales para reúso de aguas residuales en agricultura. Los contaminantes emergentes identificados por ESI-MS-TOF fueron tetraciclina, cefaclor, cefadroxilo, ampicilina, clonazepam, lormetazepam, secobarbital, maprotilina, levotiroxina, cis-androsterona, paracetamol, lidocaína, bromfeniramina, fexofenadina, amfetamina, morfina, benzoilecgonina, 11-nor- $\Delta 9-\mathrm{THC}-9-\mathrm{COOH}$, dimetilamfetamina, fenciclidina, metadona y polietilenglicol. La eficiencia de remoción de estos contaminantes en los efluentes fue de $25.8 \%$, con extremos de 0 y $74.5 \%$ para el polietilenglicol y metadona, respectivamente.

Key words: pharmaceutical products, irrigation water, trace metals, agricultural reuse 


\begin{abstract}
In Mexico, $54 \%$ of the wastewater generated is not treated and it is discharged into the water bodies, soils and irrigation canals, leading to a severe pollution problem. This represents a high risk to human health and aquatic biota. In recent decades, emerging contaminants have been detected in surface water in contact with raw and treated sewage. These compounds and their reactive metabolites cause severe toxicological effects on aquatic organisms and soil microorganisms even at low concentrations. The objective of this work was to analyze the physical and chemical properties: $\mathrm{pH}$, electrical conductivity (EC), total dissolved solids (TDS), $\mathrm{Ca}^{2+}, \mathrm{Mg}^{2+}, \mathrm{Na}^{+}$, boron (B), total phosphorus $(\mathrm{P})$ and trace metals $(\mathrm{Mn}, \mathrm{Zn}, \mathrm{Fe}$ and $\mathrm{Cu}$ ), and to identify and quantify emerging contaminants (pharmaceuticals and drugs of abuse) in the influent and effluent of Wastewater Treatment Plant (WWTP) of Morelia, using standardized methodologies, such as infrared spectroscopy FT-IR and mass spectrometry ESI-TOFMS. The values of $\mathrm{pH}, \mathrm{EC}, \mathrm{TDS}$, sodium adsorption ratio (SAR), B, P and trace metals were within the limits set by Mexican standards and international guidelines for wastewater reuse in agriculture. Emerging contaminants identified by ESI-TOF-MS were tetracycline, cefaclor, cefadroxil, ampicillin, clonazepam, lormetazepam, secobarbital, maprotiline, levothyroxine, cis-androsterone, paracetamol, lidocaine, brompheniramine, fexofenadine, amphetamine, morphine, benzoylecgonine, 11-nor- $\triangle 9$-THC-9-COOH, dimetilamfetamina, phencyclidine, methadone y polyethyleneglycol. The removal efficiency of these pollutants in the effluent was $25.8 \%$, with extremes of 0 and $74.5 \%$ for polyethyleneglycol and methadone, respectively.
\end{abstract}

\section{INTRODUCCIÓN}

Actualmente uno de los desafíos más grandes a nivel mundial es garantizar la calidad de los recursos hídricos, dado que cotidianamente se vierten a los ecosistemas acuáticos desechos industriales, urbanos y productos utilizados para el bienestar humano a través de las aguas residuales. En México, el 54 \% de las aguas residuales no son tratadas (CONAGUA 2015) y en esas condiciones son utilizadas en el riego agrícola o son vertidas directamente a los cuerpos de agua causando un severo problema de contaminación. La generación constante de nuevos productos químicos y su uso intensivo en las actividades antrópicas está conduciendo a una mayor degradación de los recursos hídricos en todo el planeta por la adición de materia orgánica, sales solubles, metales pesados y diversas sustancias con efectos tóxicos a la biota. La materia orgánica puede ser descompuesta por procesos químicos o biológicos, mientras que los metales traza pueden permanecer por largo tiempo en el ambiente debido a que no están sujetos a este tipo de transformaciones (Cherfi et al. 2015), aunque sí puede cambiar su forma química dependiendo de factores como el $\mathrm{pH}$ y el potencial de reducciónoxidación (Eh).

En las últimas dos décadas se han identificado grupos de contaminantes que se caracterizan por una alta persistencia y baja degradación en el ambiente denominados contaminantes emergentes (Tadeo et al. 2012). Los grupos de compuestos más investigados por sus efectos adversos sobre los ecosistemas (disrupción endócrina, microorganismos resistentes a antibióticos, metabolitos reactivos) son los detergentes, los productos farmacéuticos, productos de cuidado personal, hormonas, edulcorantes, pesticidas, drogas ilícitas y sus metabolitos, así como las fragancias, los aditivos de gasolinas y los retardadores de flama. Se estima que a nivel mundial se vierten anualmente a las aguas superficiales cerca de 300 millones de toneladas de compuestos sintéticos, entre los que se encuentran productos farmacéuticos, industriales y de consumo (Kümmerer 2011). La persistencia de estos compuestos en el medio acuático depende de sus propiedades químicas como: solubilidad, volatilidad, absorción, biodegradación, polaridad y estabilidad; estos factores afectan la eficiencia de los procesos de tratamiento de las aguas residuales donde son transportados y, por ende, el grado de remoción de estos contaminantes. Las concentraciones de contaminantes como los productos farmacéuticos que se han detectado en efluentes de plantas de tratamiento varían de 0.0016 a $373 \mu \mathrm{g} / \mathrm{L}$, dependiendo de la población, ubicación y proceso de tratamiento a que son sometidas las aguas residuales (Verlicchi et al. 2012). En muchos casos estos compuestos fueron introducidos en el ambiente desde hace tiempo y no se habían detectado debido a la falta de equipos 
altamente sensibles como la cromatografía de líquidos de alta resolución (HPLC, por sus siglas en inglés) y cromatografías de gases y líquidos acopladas a masas (GC-MS y LC-MS, por sus siglas en inglés).

La espectroscopia de infrarrojo (IR) con transformada de Fourier (FT, por sus siglas en inglés) es una técnica cualitativa que permite identificar de manera rápida compuestos desconocidos, con menor ruido analítico y mayor sensibilidad óptica en relación con instrumentos IR que no tienen integrado el algoritmo FT. Esta técnica se utiliza frecuentemente en ciencias forenses para la identificación de drogas y tóxicos y se fundamenta en la comparación de los espectros de las muestras con los espectros de referencia (bibliotecas) mediante un coeficiente de correlación (Moffat et al. 2011). Si bien en mezclas complejas pueden presentarse interferencias que dan lugar a una superposición de los espectros de los compuestos individuales, dificultando la separación de espectros individuales y su identificación, los modernos espectrómetros FT-IR permiten obtener información general sobre los compuestos existentes en este tipo de matrices.

La espectrometría de masas (MS, por sus sigles en inglés) es de amplio uso en el análisis cuantitativo de contaminantes emergentes. La separación y determinación de iones por esta técnica se basa en la formación de moléculas con carga (iones moleculares) y el análisis de su relación masa/carga $(\mathrm{m} / \mathrm{z})$. La principal limitación de la técnica es la fragmentación de los iones que se genera cuando las moléculas son sometidas a ionización altamente energética, lo que eleva el ruido analítico. Sin embargo, la fragmentación de iones depende en gran medida del método de ionización y el tipo de detector de iones integrado en el equipo MS. La ionización por electronebulización (ESI, por sus siglas en inglés) genera poca fragmentación y el detector del tipo tiempo de vuelo (TOF, por sus siglas en inglés) tiene alta sensibilidad para detectar grandes biomoléculas (Anderson et al. 2004, Silverstein et al. 2005). Las técnicas más modernas incorporan separaciones cromatográficas acopladas a espectrometría de masas (GC-MS y LC-MS), pero sus costos son elevados. Las técnicas FT-IR y ESI-MS-TOF empleadas en este trabajo se seleccionaron por su uso extendido en el análisis de matrices complejas dentro de las ciencias forenses (Moffat et al. 2011). Si bien en este trabajo no se utilizaron separaciones cromatográficas o químicas, estas técnicas posibilitan un análisis panorámico de los grupos de contaminantes emergentes presentes en las aguas residuales crudas y tratadas bajo estudio, lo cual permite establecer las bases para futuras investigaciones sobre estos compuestos en la zona.
En México se tiene poca información científica sobre la presencia de mezclas complejas de contaminantes no regulados, que tienen diversos orígenes y que representan un problema ambiental. Los trabajos de Durán-Álvarez et al. (2014 y 2015) han abierto estas investigaciones en este país, centrándose en la cuantificación, transporte y biodegradación de contaminantes farmacéuticos como el naproxen, carbamacepina y triclosan. Rocha-Gutiérrez et al. (2015), en una revisión sobre contaminantes emergentes en México del tipo de los compuestos de éteres de bifenilos polibromados (PBDE, por sus sigles en inglés), mencionan que éstos se detectaron en influentes y efluentes de plantas de tratamiento en concentraciones de 40 a 215 y 3 a $91 \mu \mathrm{g} / \mathrm{L}$, respectivamente. $\mathrm{La}$ escasez de datos ambientales disponibles sobre los contaminantes emergentes dificulta su regulación a través de las normas mexicanas para el control de la polución del agua. Esto es relevante en un contexto de escasez creciente de agua dulce y donde se buscan alternativas para disponer de aguas residuales tratadas para su reutilización en la agricultura (CONAGUA 2015). Por otro lado, los sistemas de tratamiento de aguas residuales que operan en México fueron diseñados para depurar sólidos, materia orgánica disuelta y nutrientes, por lo que muchos compuestos tóxicos pasan el proceso de tratamiento con poca o ninguna modificación en su concentración.

Los objetivos de este trabajo fueron determinar las propiedades físicas y químicas, metales pesados e identificar y cuantificar los contaminantes emergentes (farmacéuticos y drogas de abuso), en los influentes y efluentes de la Planta de Tratamiento de Aguas Residuales de la ciudad de Morelia, Michoacán, México, y analizar el potencial de reúso en riego agrícola de estos flujos.

\section{MATERIALES Y MÉTODOS}

\section{Sitio de estudio}

Se obtuvieron muestras de aguas residuales del influente y del efluente de la Planta de Tratamiento de Aguas Residuales de Morelia, Michoacán, México (PTAR-Atapaneo). La población servida es de 597 511 hab. La PTAR-Atapaneo tiene 8 años de operación y recibe aguas residuales (AR) domésticas e industriales (papelera, concreto, aceros, alimenticia) que se mezclan con aguas pluviales en temporada de lluvias. El proceso de tratamiento de las AR comprende 8 etapas: 1) tratamiento primario (cribado), 2) sedimentación primaria, 3) filtros biológicos, 4) reactor biológico (lodos activados), 5) sedimentador 
secundario, 6) unidad de desinfección (cloración), 7) unidad de recirculación, y 8) tratamiento de lodos (secado por centrifugación y temperatura). Tiene una capacidad instalada de $1200 \mathrm{~L} / \mathrm{s}$ de influentes, con una DBO (demanda bioquímica de oxígeno) promedio de $273 \mathrm{mg} / \mathrm{L}$ (información proporcionada por el personal técnico, PTAR-Atapaneo).

\section{Muestreo}

El muestreo se realizó del 24 al 30 de junio de 2013 (temporada de estiaje) para evitar la dilución de contaminantes con las lluvias. Se tomó $1 \mathrm{~L}$ del influente y $1 \mathrm{~L}$ del efluente cada hora durante $24 \mathrm{~h}$. Posteriormente se colectó una muestra diaria (influente y efluente) a la misma hora durante seis días para hacer un total de 60 muestras. Las muestras se almacenaron en contenedores ámbar a una temperatura de 2 a $4{ }^{\circ} \mathrm{C}$ hasta su análisis.

\section{Análisis físicos y químicos}

En campo se determinaron: $\mathrm{pH}$, conductividad eléctrica (CE) y sólidos disueltos totales (SDT) Hanna Instruments HI9635. En el laboratorio se analizaron $\mathrm{Ca}^{2+}, \mathrm{Mg}^{2+}, \mathrm{Na}^{+}, \mathrm{B}$ (azometina), $\mathrm{P}$ (persulfato) y metales pesados totales (Mn, Zn, Fe y $\mathrm{Cu}$, Espectrofotómetro AA 3100 Perkin Elmer) utilizando metodologías estandarizadas (Eaton et al. 2005, Rodier et al. 2011). La relación de adsorción de sodio (RAS), como indicador del potencial de sodificación de los suelos por el uso de estas aguas en el riego agrícola (Ayers y Westcot 1994), se calculó con la fórmula:

$R A S=\frac{N a}{\sqrt{\frac{C a+M g}{2}}}$

Donde $R A S=$ Relación de adsorción de sodio; $\mathrm{Na}^{+}, \mathrm{Ca}^{2+}, \mathrm{Mg}^{2+}=$ concentración de sodio, calcio y magnesio en meq/L.

\section{Extracción de contaminantes emergentes Reactivos y estándares de referencia}

Los estándares de referencia fueron tetraciclina, ampicilina, cefaclor, cefadroxilo, lidocaína, paracetamol, fexofenadina, bromfeniramina, clonazepam, lormetazepam, maprotilina, androsterona, levotiroxina, polietilenglicol (Sigma-Aldrich), benzoilecgonina, metadona, d-metanfetamina, morfina, 11-nor- $\Delta 9$ THC-9-COOH, fenciclidina, secobarbital (Siemens Siva). El acetonitrilo, agua, ácido fórmico, metanol y cloroformo utilizados fueron grado HPLC (SigmaAldrich, México).
Se usó una extracción líquido-líquido (RoblesMolina et al. 2010, Stimpfl 2011). Se tomó un volumen de $600 \mathrm{~mL}$ de cada muestra de agua y se separó en porciones de $200 \mathrm{~mL}$ para llevar a distintos valores de $\mathrm{pH}(4.0,7.0$ y 10.0$)$, empleando $\mathrm{HCl} 1 \mathrm{Ny} \mathrm{NH}_{4} \mathrm{OH}$ $1.5 N$ ). Cada porción se mezcló con cloroformo (relación 1:1) en un embudo de separación, agitando por 15 minutos. Se dejó reposar la mezcla y se colectó la fase clorofórmica en un matraz; el procedimiento se repitió tres veces con la misma muestra. Enseguida se evaporó la muestra casi a sequedad en un evaporador rotatorio de vacío (Buchi R II), equipado con baño de calentamiento. Las condiciones de evaporación fueron: $38^{\circ} \mathrm{C}$ y presión de 474 mbar. Un testigo con cloroformo fue tratado de la misma manera, esto para detectar posibles contaminantes emergentes en el solvente. Los extractos fueron disueltos en $2 \mathrm{~mL}$ de metanol y almacenados en frascos ámbar a $-20{ }^{\circ} \mathrm{C}$ hasta su análisis. En total se obtuvieron 180 extractos.

\section{Análisis por espectroscopía de infrarrojo (FT-IR)}

Se tomó una alícuota de cada uno de los 180 extractos (sin evaporación previa), se agregó $200 \mu \mathrm{L}$ de cloroformo y se analizaron en un espectrómetro de infrarrojo FT-IR Perkin Elmer Spectrum modelo RX 1, con celda de seleniuro de cinc. El intervalo de análisis fue de 4000 a $652 \mathrm{~cm}^{-1}$ (infrarrojo intermedio) ajustando la transmitancia $\mathrm{T}$ a $100 \%$. Los datos fueron comparados con la biblioteca FT-IR Perkin Elmer Spectrum incluida en el espectrómetro. Posteriormente los extractos fueron nuevamente almacenados en metanol a $-20{ }^{\circ} \mathrm{C}$ para su análisis por espectrometría de masas (ESI-MS-TOF).

\section{Análisis por espectrometría de masas (ESI-MS- TOF)}

Para este análisis se seleccionaron al azar 78 extractos (13 extractos de influentes y 13 extractos de efluentes, correspondientes a la misma hora de muestreo, y a pH 4.0, 7.0 y 10.0). Se usó la tetraciclina como estándar interno para corregir efectos de matriz durante el análisis (Stimpfl 2011). Los extractos seleccionados fueron reconstituidos, sin evaporación previa, en una solución de metanolacetonitrilo 1:1 (fase móvil B) y solución de ácido fórmico $0.1 \%$ (fase móvil A). En el análisis se utilizó el modo de ionización ESI-positivo, en un rango de pesos moleculares entre 50 y $900 \mathrm{~g} / \mathrm{mol}$. El sistema de detección de masas utilizado fue de tiempo de vuelo MicroOTOF QII Bruker Daltonics, con la fuente de ionización por electroespray ESI. Los parámetros de masas elegidos fueron: voltaje de capilar $2700 \mathrm{~V}$, fuente de iones a $180{ }^{\circ} \mathrm{C}$, nitrógeno a $4.0 \mathrm{~L} / \mathrm{min}$ y 
40 psi. Para obtener el ajuste de la curva y el cálculo de las concentraciones se usaron concentraciones crecientes de los estándares de referencia $(1,10,15$, 20 y $200 \mu \mathrm{g} / \mathrm{L}$ ).

Se calculó la eficiencia de remoción de los contaminantes emergentes detectados en la PTAR mediante la relación:

$E=100-\left[(\mathrm{Ce} / \mathrm{Ci})^{*} 100\right]$

\section{Donde:}

$E=$ Eficiencia de remoción; $C e=$ concentración del compuesto en el efluente; $C i=$ concentración del compuesto en el influente.

\section{RESULTADOS Y DISCUSIÓN}

\section{Análisis físicos y químicos}

Los parámetros evaluados en las muestras obtenidas de las aguas residuales (influentes y efluentes de la PTAR), se presentan en el cuadro I. En los influentes se detectaron los metales $\mathrm{Cu}, \mathrm{Zn}, \mathrm{Fe}, \mathrm{Mn}$ en concentraciones $<1$ a $53.8 \mathrm{mg} / \mathrm{L}$. El orden de concentración media fue: $\mathrm{Fe}>\mathrm{Mn}>\mathrm{Zn}>\mathrm{Cu}$. Diversos trabajos han mostrado que los metales Fe, Zn, Mn y $\mathrm{Cu}$ están entre los más abundantes en las aguas residuales sin tratar (Karvelas et al. 2003, Cherfi et al. 2015). Los parámetros $\mathrm{Cu}$ y $\mathrm{Zn}$ de los efluentes cumplen con la Norma Mexicana NOM-001-ECOL-1996 (SEMARNAT 1997) en relación con los límites máximos permisibles de contaminantes para descargas de aguas residuales en ríos y embalses naturales y artificiales para agua de usos agrícola, público urbano y de protección a la vida acuática. La norma no establece los límites para metales como Fe y Mn (SEMARNAT 1997). Otras normativas mencionan como límites máximos permitidos concentraciones de 40.0 y $4.0 \mathrm{mg} / \mathrm{L}$ para Fe y $\mathrm{Mn}$, respectivamente, en aguas residuales tratadas (Karvelas et al. 2003). La concentración máxima de estos metales en los efluentes analizados se encuentra por debajo de estos límites. Se desconoce si los efluentes presentan otros elementos tóxicos como $\mathrm{Cd}, \mathrm{Cr}, \mathrm{Pb}, \mathrm{Hg}$ y $\mathrm{As}$ que incluye la NOM-001-ECOL-1996. La concentración de $\mathrm{P}$ total no excede el límite marcado por la NOM-001-ECOL-1996 (20 mg/L) al igual que el pH (rango: 5 a 10). En los efluentes la CE y el $\mathrm{B}$ se redujeron en 13 y $33 \%$, respectivamente, en comparación con los influentes; la RAS no mostró variación indicando que la relación entre los cationes solubles $\mathrm{Ca}^{2+}, \mathrm{Mg}^{2+}$ y $\mathrm{Na}^{+}$no fue afectada por el proceso de tratamiento en la PTAR. La baja eficiencia para reducir las sales solubles es característica de las PTAR que utilizan procesos primarios y secundarios (lodos activados), requiriéndose para aumentarla el empleo de procesos terciarios como membranas de intercambio iónico, ósmosis inversa o ultrafiltración, entre otros (Kornboonraksa 2016). Por otro lado, los valores de CE, B y RAS muestran que los efluentes analizados tienen un grado de restricción de leve a moderado para usarse en el riego de cultivos, en

CUADRO I. VALORES DE LOS PARÁMETROS FÍSICOS Y QUÍMICOS Y METALES TRAZA EN LOS INFLUENTES Y EFLUENTES DE LA PTAR ATAPANEO

\begin{tabular}{|c|c|c|c|c|c|c|c|c|}
\hline \multirow{2}{*}{ Parámetro } & \multicolumn{3}{|c|}{ Influente } & \multicolumn{3}{|c|}{ Efluente } & \multirow[b]{2}{*}{ Media } & \multirow{2}{*}{ - Valores de referencia } \\
\hline & Unidad & Máximo & Mínimo & Media & Máximo & Mínimo & & \\
\hline $\mathrm{pH}$ & & 7.8 & 6.8 & 7.1 & 7.4 & 6.4 & 7.0 & $6.5-8.4^{*}$ \\
\hline $\mathrm{CE}$ & $\mu \mathrm{S} / \mathrm{cm}$ & 917 & 454 & 710 & 688 & 588 & 618 & $200-700$ (restricción leve o moderada) * \\
\hline SDT & $\mathrm{mg} / \mathrm{L}$ & 910 & 227 & 372 & 345 & 294 & 309 & \\
\hline $\mathrm{Ca}$ & $\mathrm{meq} / \mathrm{L}$ & 2.0 & 1.4 & 1.8 & 1.5 & 1.3 & 1.4 & \\
\hline $\mathrm{Mg}$ & $\mathrm{meq} / \mathrm{L}$ & 1.3 & 0.5 & 0.9 & 1.0 & 0.6 & 0.7 & \\
\hline $\mathrm{Na}$ & meq/L & 4.6 & 1.9 & 3.5 & 3.9 & 2.6 & 3.1 & \\
\hline B & mg/L & 1.3 & 0.3 & 0.6 & 0.8 & nd & 0.4 & $<0.7$ (sin restricción)* \\
\hline $\mathrm{P}$ & $\mathrm{mg} / \mathrm{L}$ & 5.6 & 0.4 & 1.6 & 2.6 & nd & 1.0 & $10-20 * *$ \\
\hline RAS & & 3.9 & 2.3 & 3.1 & 3.9 & 2.6 & 3.1 & $\begin{array}{l}0 \text { - } 3 \text { (restricción leve a moderada, para CE de } \\
200 \text { a } 700 \mu \mathrm{S} / \mathrm{cm})^{*}\end{array}$ \\
\hline $\mathrm{Mn}$ & $\mathrm{mg} / \mathrm{L}$ & 1.1 & 0.3 & 0.5 & 0.3 & $\mathrm{nd}^{* * *}$ & 0.1 & $0.2^{*}$ \\
\hline $\mathrm{Zn}$ & $\mathrm{mg} / \mathrm{L}$ & 1.2 & nd & 0.3 & 0.3 & nd & 0.2 & $2.0^{*}$ \\
\hline $\mathrm{Fe}$ & $\mathrm{mg} / \mathrm{L}$ & 53.8 & nd & 8.7 & 1.2 & nd & 0.5 & $5.0^{*}$ \\
\hline $\mathrm{Cu}$ & $\mathrm{mg} / \mathrm{L}$ & 1.1 & nd & 0.1 & 0.1 & nd & 0.01 & $0.2 *$ \\
\hline
\end{tabular}

$\mathrm{CE}=$ conductividad eléctrica, $\mathrm{SDT}=$ sólidos disueltos totales, RAS $=$ relación de adsorción de sodio. * (Ayers y Westcot 1994), ** Uso agrícola (SEMARNAT 1997); *** nd, no detectado; concentración menor al límite de detección 
tanto que la concentración de los metales $\mathrm{Cu}, \mathrm{Zn}, \mathrm{Fe}$ y Mn no es restrictiva para este uso (Ayers y Westcot 1994). Estos efluentes pueden reutilizarse en el riego agrícola en suelos de buen drenaje, donde las sales que se adicionan con el agua de riego se lixivian reduciendo su acumulación en el perfil del suelo. En suelos con drenaje deficiente el riego con este tipo de flujos requiere medidas de manejo especiales para reducir los problemas de acumulación de sales que pueden afectar negativamente los rendimientos de los cultivos. La utilización de aguas residuales tratadas en el riego agrícola requiere, además, una medición periódica de los parámetros de fertilidad y calidad del suelo (Idowu et al. 2015).

En los efluentes los metales traza (Cu, Zn, Fe, Mn) se redujeron en $92,36,95$ y $71 \%$, respectivamente, en relación con su concentración en los influentes y no rebasaron los límites para el uso de estas aguas en riego agrícola. Diversos estudios han mostrado la adaptabilidad de los efluentes de aguas residuales tratadas en el riego de hortalizas debido a la reducción significativa de contaminantes orgánicos, microbiológicos y metales pesados que se observa (Cherfi et al. 2015). Sin embargo, estos metales se acumulan en los lodos residuales y si estos son depositados al aire libre, sin un tratamiento adecuado, pueden causar un severo problema de contaminación ambiental por su contenido de patógenos, metales pesados y productos farmacéuticos (Kim et al. 2015, Verlicchi y Zambello 2015).

\section{Contaminantes emergentes}

Espectroscopía de infrarrojo FT-IR. Los 180 espectros obtenidos se compararon con los de la Biblioteca Perkin Elmer Spectrum, integrada al programa del equipo FT-IR utilizado y se seleccionaron aquellos que presentaron una correlación $(\mathrm{R})$ $\geq 0.80$ con los espectros de referencia (Fig. 1 a 6 ). Los espectros seleccionados correspondieron a seis grupos de productos farmacéuticos: 1) antibióticos
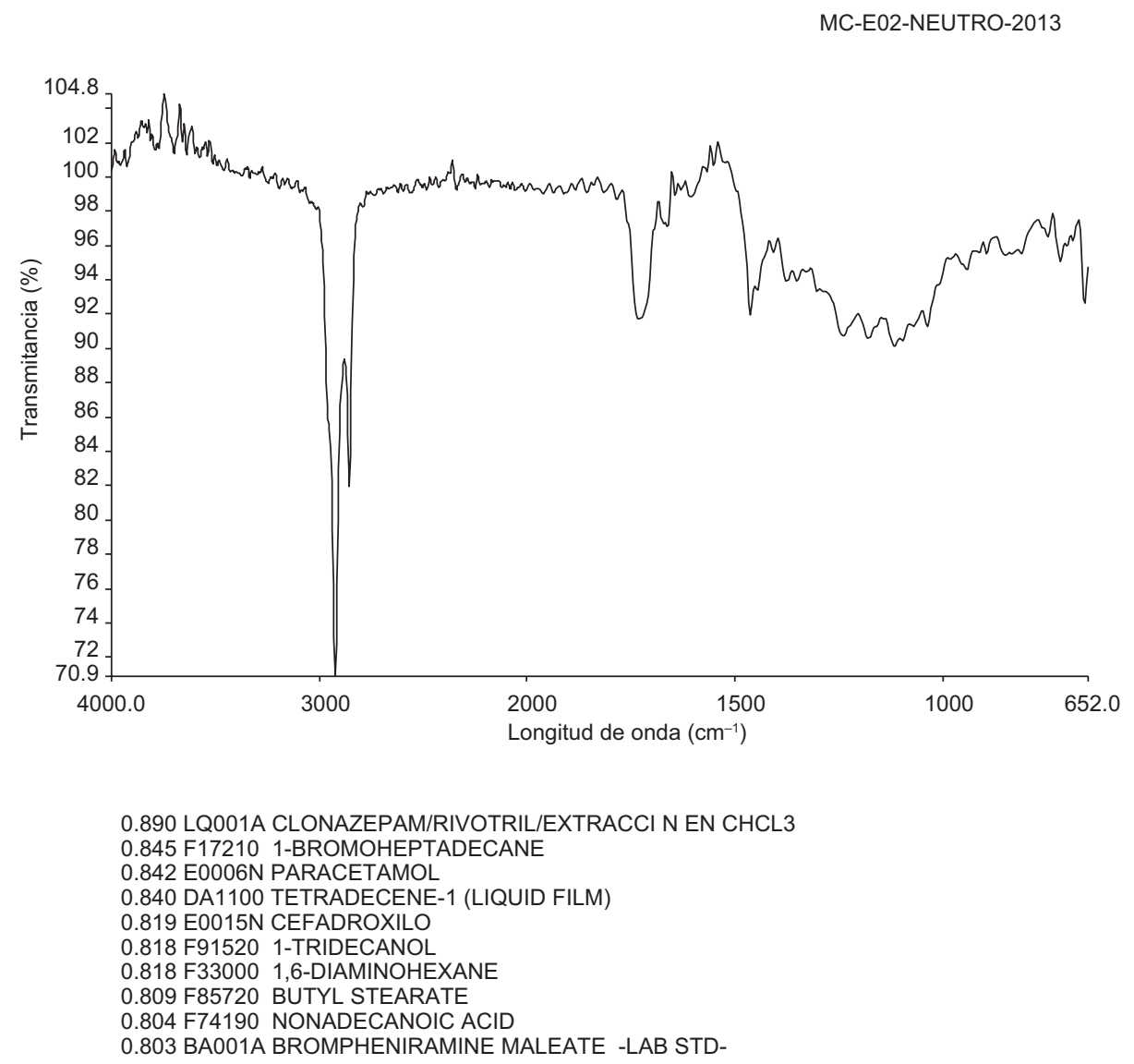

Fig. 1. Espectro obtenido en instrumento de infrarrojo con transformada de Fourier y principales compuestos identificados a $\mathrm{pH}=7.0$. Aguas residuales tratadas. Productos farmacéuticos de interés: clonazepam $(\mathrm{R}=0.890)$, paracetamol $(\mathrm{R}=0.842)$, cefadroxilo $(\mathrm{R}=0.819)$, maleato de bromfeniramina $(\mathrm{R}=0.803)$ 


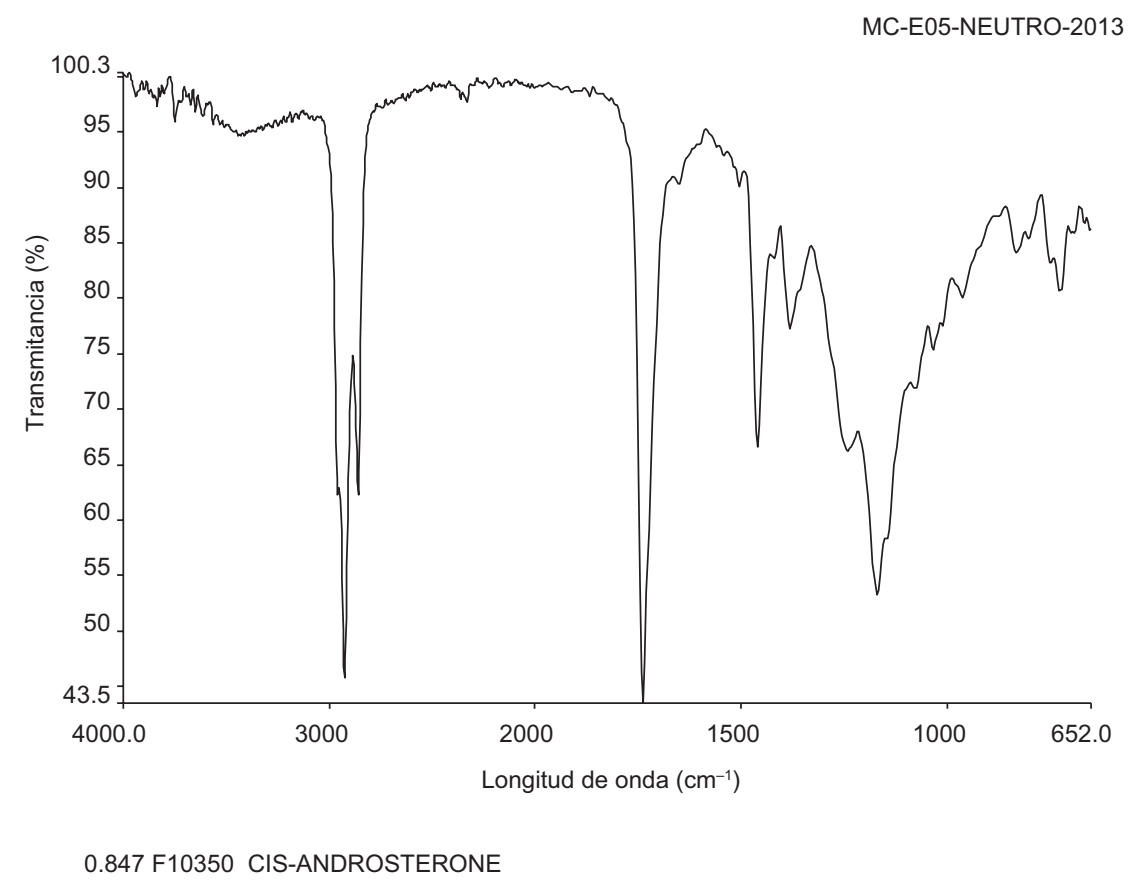

Fig. 2. Espectro obtenido en instrumento de infrarrojo con transformada de Fourier y principales compuestos identificados a $\mathrm{pH}=7.0$. Aguas residuales tratadas. Productos farmacéuticos de interés: cis-androsterona $(\mathrm{R}=0.847)$

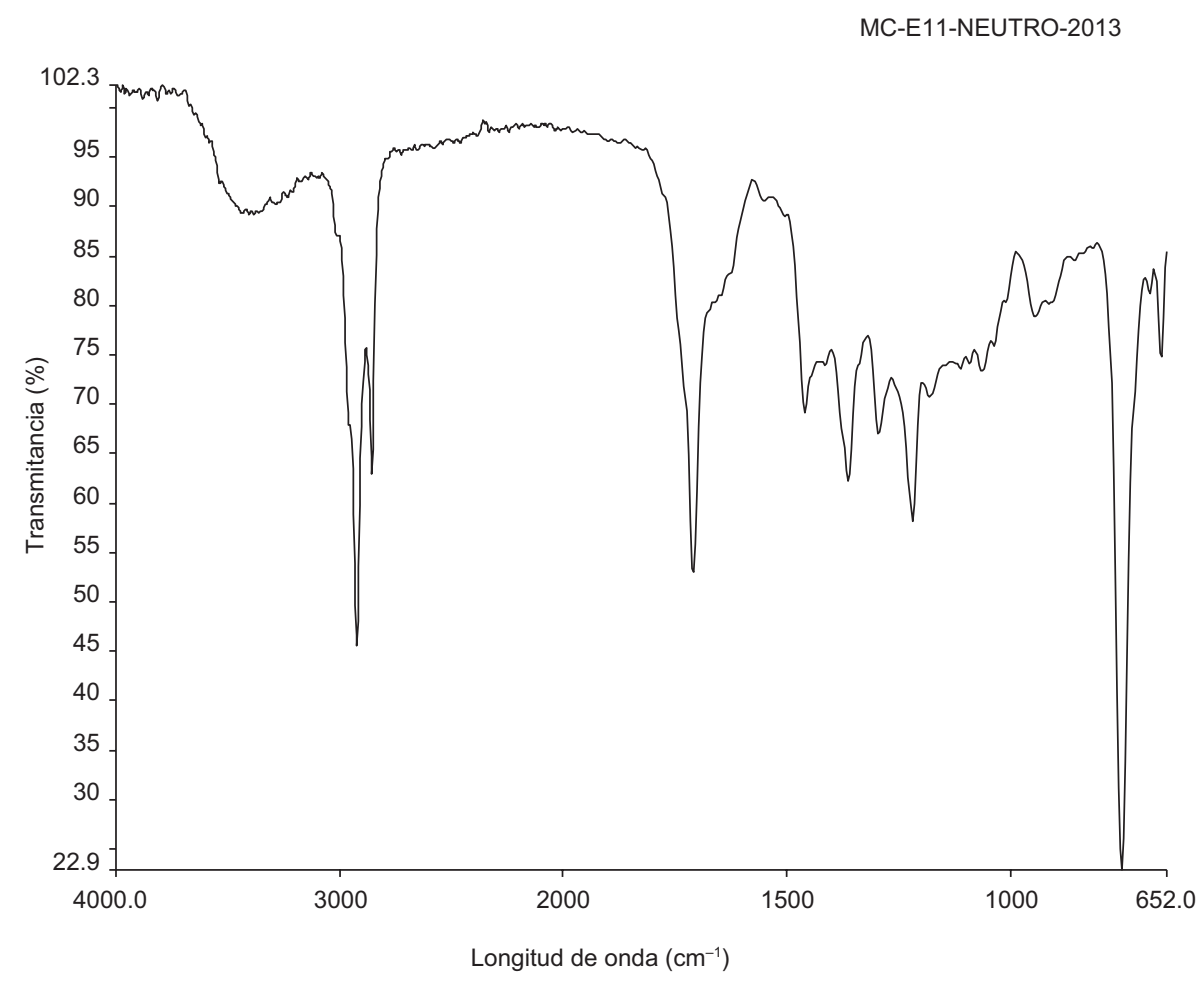

0.814 MC012E LUDIOMIL: CLORHIDRATO DE MAPROTILINA (DIBENZODIAZEPINA).

Fig. 3. Espectro obtenido en instrumento de infrarrojo con transformada de Fourier y principales compuestos identificados a $\mathrm{pH}=7.0$. Aguas residuales tratadas. Productos farmacéuticos de interés: clorhidrato de maprotilina $(\mathrm{R}=0.814)$ 


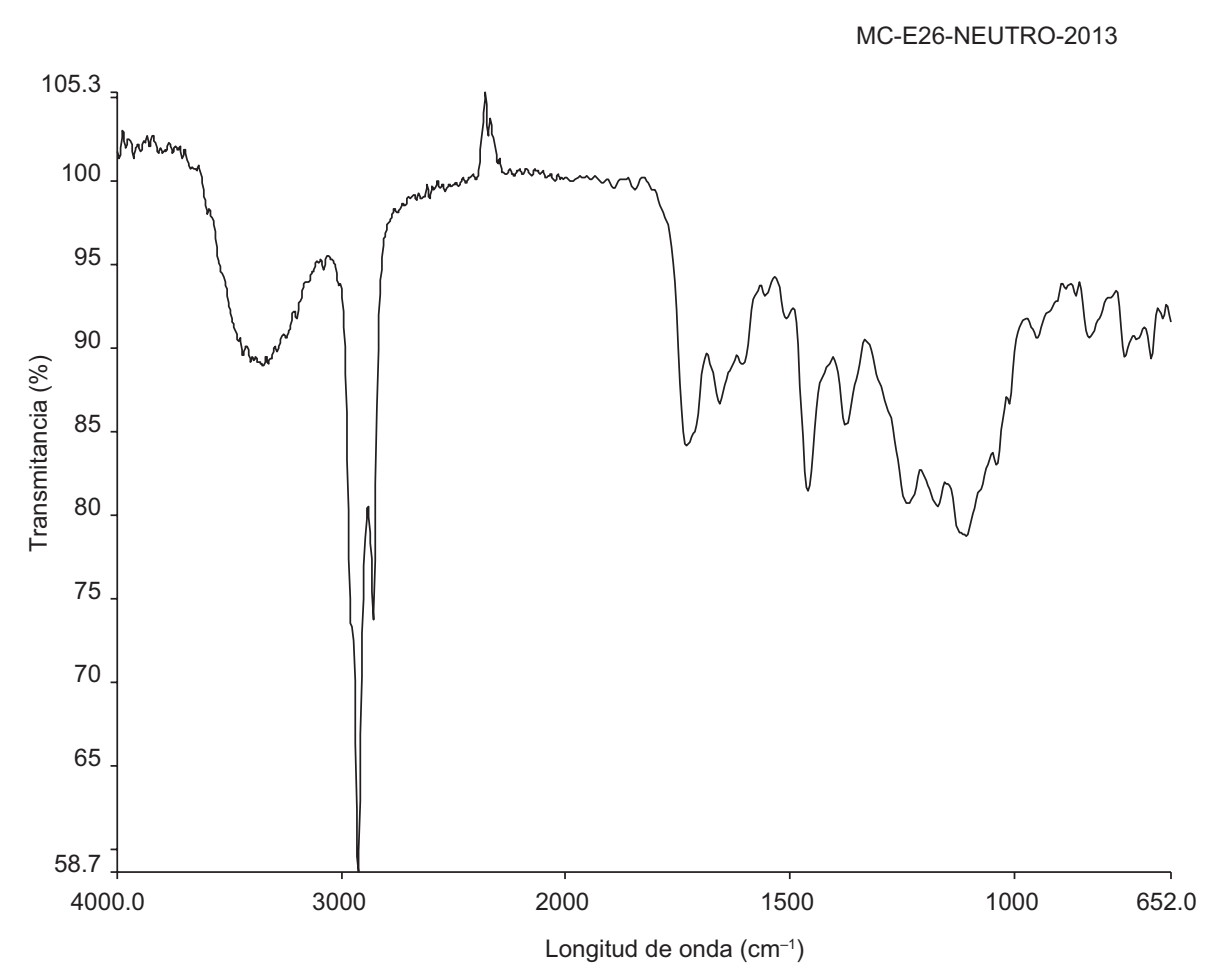

0.832 LQ001A CLONAZEPAM/RIVOTRIL/EXTRACCI N EN CHCL3

0.829 E0015N CEFADROXILO

0.807 F85720 BUTYL STEARATE

0.804 DA1168 POLYETHYLENE OXIDIZED ACID NUMBER 15MG KOH/G/(KBR FILM)/SP-56

0.802 E0017N LEVOTIROXINA

Fig. 4. Espectro obtenido en instrumento de infrarrojo con transformada de Fourier y principales compuestos identificados a $\mathrm{pH}=7.0$. Aguas residuales tratadas. Productos farmacéuticos de interés: clonazepam $(\mathrm{R}=0.832)$, cefadroxilo $(\mathrm{R}=0.829)$, levotiroxina $(\mathrm{R}=0.802)$

(cefadroxilo, cefaclor), 2) analgésicos (paracetamol), 3) anestésicos (lidocaína), 4) antihistamínicos (bromfeniramina), 5) ansiolíticos (clonazepam, maprotilina) y 6) hormonas y esteroides (levotiroxina, cis-androsterona). No se detectaron por esta técnica drogas de abuso. La metodología FT-IR identifica compuestos con base en la rotación/vibración que sufren las moléculas como consecuencia de la absorción de radiación (en este caso, en el infrarrojo medio o región de la "huella dactilar"). En muestras complejas se encuentran distintos tipos de átomos y enlaces y se genera una gran cantidad de vibraciones posibles que dificultan el análisis de los espectros resultantes; la identificación de moléculas, en este caso, requiere una purificación previa como la separación cromatográfica (Jee 2011). Estas características de FT-IR explican el reducido número de compuestos detectados por esta metodología, la cual se usa principalmente en el análisis cualitativo.
Espectrometría de masas ESI-MS-TOF. En el cuadro II se muestran las características y la frecuencia de los contaminantes emergentes detectados, tanto en el influente como en el efluente. La frecuencia se calculó como el porcentaje de las muestras donde se detectó el compuesto en relación con el total de muestras analizadas $(\mathrm{n}=78)$. Se identificaron 22 compuestos correspondientes a ocho grupos: 1) antibióticos (tetraciclina, cefaclor, cefadroxilo, ampicilina), 2) antidepresivos y ansiolíticos (clonazepam, lormetazepam, secobarbital, maprotilina), 3) hormonas y esteroides (levotiroxina, cis-androsterona), 4) analgésicos (paracetamol), 5) anestésicos (lidocaína), 6) antihistamínicos (bromfeniramina, fexofenadina), 7) drogas de abuso (amfetamina, morfina, benzoilecgonina, 11-nor- $\Delta$ 9-THC-9-COOH, dimetilamfetamina, fenciclidina, metadona) y 8) otros (polietilenglicol). Estos resultados confirman lo encontrado con la metodología FT-IR donde se detectaron nueve de estos compuestos. 
MC-E17-ACIDO-2013

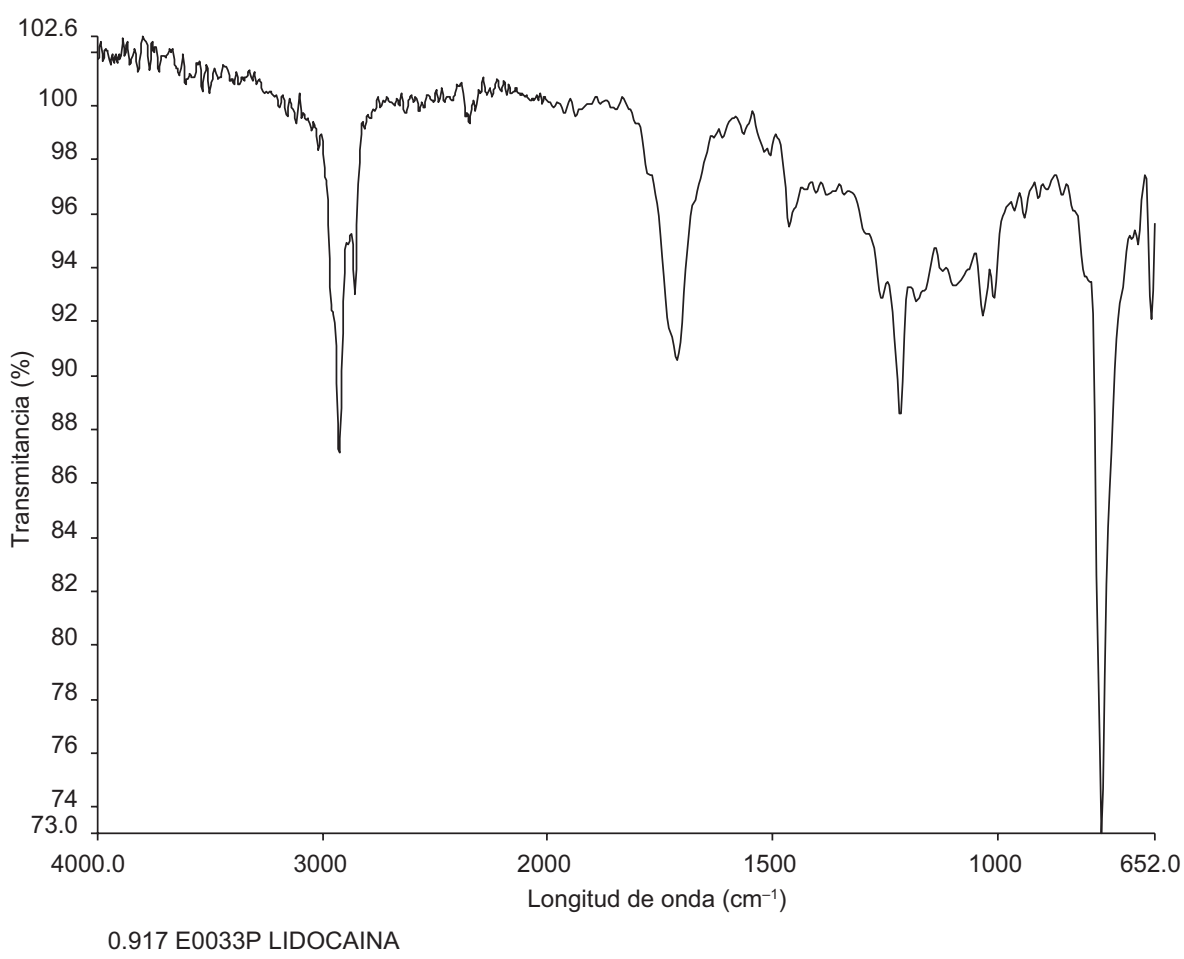

Fig. 5. Espectro obtenido en instrumento de infrarrojo con transformada de Fourier y principales compuestos identificados a $\mathrm{pH}=4.0$. Aguas residuales tratadas. Productos farmacéuticos de interés: lidocaína $(\mathrm{R}=0.917)$

MC-E17-ALCALINO-2013

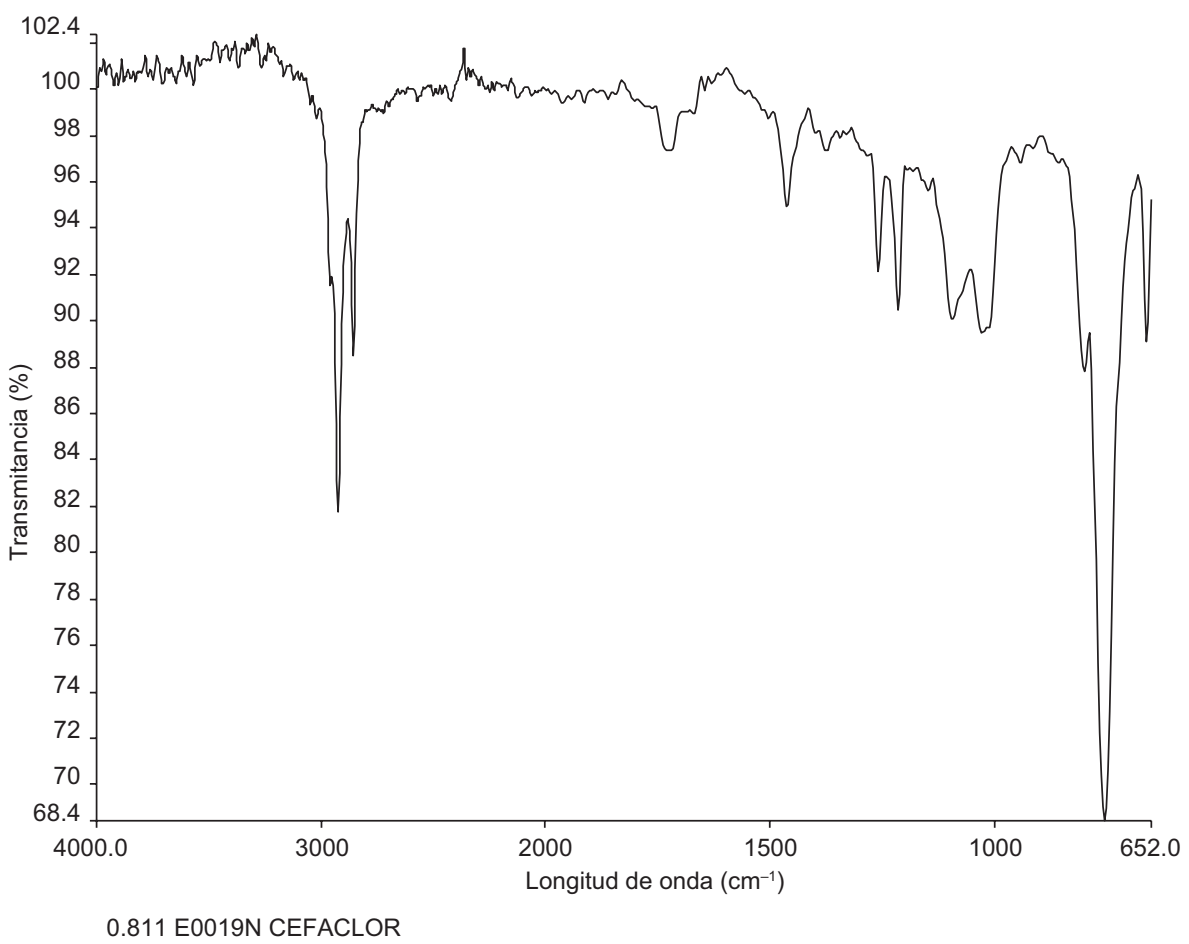

Fig. 6. Espectro obtenido en instrumento de infrarrojo con transformada de Fourier y principales compuestos identificados a $\mathrm{pH}=10$. Aguas residuales tratadas. Productos farmacéuticos de interés: cefaclor $(\mathrm{R}=0.811)$ 
CUADRO II. CARACTERÍSTICAS Y FRECUENCIA DE LOS CONTAMINANTES EMERGENTES DETECTADOS POR ESPECTROMETRÍA DE MASAS CON IONIZACIÓN POR ELECTRONEBULIZACIÓN Y DETECTOR DE TIEMPO DE VUELO (ESI-MS-TOF)

\begin{tabular}{lcccc}
\hline Compuesto & $\mathrm{m} / \mathrm{z}$ teórico* & $\mathrm{m} / \mathrm{z}$ analizado** & $\begin{array}{c}\text { Frecuencia en } \\
\text { el influente }(\%)\end{array}$ & $\begin{array}{c}\text { Frecuencia en } \\
\text { el efluente }(\%)\end{array}$ \\
\hline Tetraciclina & 444.5 & 445.5 & 92.3 & 84.6 \\
\hline Cefaclor & 385.8 & 408.8 & 76.9 & 69.2 \\
Cefadroxilo & 363.4 & 386.2 & 61.5 & 61.5 \\
Ampicilina & 349.4 & 350.4 & 100.0 & 100.0 \\
Clonazepam & 315.7 & 338.7 & 15.4 & 15.4 \\
Lormetazepam & 327.2 & 328.2 & 100.0 & 100.0 \\
Secobarbital & 238.3 & 240.3 & 53.8 & 38.5 \\
Maprotilina & 277.4 & 284.2 & 69.2 & 69.2 \\
Levotiroxina & 776.9 & 799.6 & 30.8 & 30.8 \\
Cis-androsterona & 290.4 & 291.3 & 69.2 & 61.5 \\
Paracetamol & 151.2 & 174.1 & 61.5 & 46.2 \\
Lidocaína & 234.3 & 235.3 & 69.2 & 69.2 \\
Bromfeniramina & 319.2 & 342.2 & 70.0 & 70.0 \\
Fexofenadina & 501.7 & 502.7 & 100.0 & 100.0 \\
Amfetamina & 135.2 & 136.1 & 100.0 & 53.8 \\
Morfina & 303.4 & 304.4 & 84.6 & 46.2 \\
Benzoilecgonina & 289.3 & 290.2 & 30.7 & 15.4 \\
11-nor- $\Delta 9-T H C-9-C O O H$ & 314.5 & 315.5 & 76.9 & 61.5 \\
Dimetilamfetamina & 163.3 & 181.1 & 69.2 & 53.8 \\
Fenciclidina & 243.4 & 260.2 & 30.8 & 30.8 \\
Metadona & 309.5 & 310.5 & 15.4 & 15.4 \\
Polietilenglicol & 445.2 & --- & 100.0 & 100.0 \\
\hline
\end{tabular}

$* \mathrm{~m} / \mathrm{z}$ teórico $=$ valor de referencia de la relación masa/carga del ion principal del analito estudiado, $* * \mathrm{~m} / \mathrm{z}$ analizado = valor obtenido de la relación masa/carga en el espectrómetro de masas (MS) del ion principal del analito estudiado.

El cuadro III resume los resultados de la cuantificación por el análisis de espectrometría de masas ESI-MS-TOF para los contaminantes emergentes detectados. Las concentraciones de los compuestos identificados en cada extracto a $\mathrm{pH}$ 4.0, 7.0 y 10.0 se sumaron, obteniéndose 13 valores para calcular el rango y el promedio de concentraciones en influentes y efluentes. El polietilenglicol fue el compuesto detectado en mayor concentración media tanto en influentes como efluentes $(135 \mu \mathrm{g} / \mathrm{L})$. En los efluentes, la tetraciclina, ampicilina y lormetazepam fueron los productos farmacéuticos de mayor concentración media $(64.9,15.5$ y $12.2 \mu \mathrm{g} / \mathrm{L}$, respectivamente). Considerando la concentración media en los influentes, se observó que solamente los compuestos secobarbital, cis-androsterona y paracetamol fueron menores a los valores de referencia reportados por otros autores (Daughton 2014, Bizkarguenaga et al. 2012, Rivera et al. 2013), mientras que la tetraciclina estuvo dentro del rango reportado ( Man-Hong et al. 2015) y 15 compuestos excedieron los valores de referencia, con extremos de 2.4 veces para la metadona y 1007 veces para la ampicilina; no se encontraron reportes sobre valores de referencia para levotiroxina, fenciclidina y polietilenglicol. Como grupo de compuestos, los antibióticos presentaron las mayores concentraciones medias en los efluentes $(21.8 \mu \mathrm{g} / \mathrm{L})$, seguidos por los antihistamínicos $(9.1 \mu \mathrm{g} / \mathrm{L})$, ansiolíticos $(4.2 \mu \mathrm{g} / \mathrm{L})$, hormonas y esteroides $(4.0 \mu \mathrm{g} / \mathrm{L})$ y drogas de abuso $(2.4 \mu \mathrm{g} / \mathrm{L})$. Los antibióticos es el grupo de medicamentos que preferentemente ingieren los consumidores en México (PROFECO 2010) y que hasta hace poco tiempo podían adquirirse libremente en las farmacias. Esta condición y el hecho de que en la metodología utilizada (ESI-MS-TOF) no se incluyó una separación cromatográfica, podrían explicar las altas concentraciones detectadas. No obstante, los datos obtenidos en este trabajo permiten un análisis panorámico de los grupos de contaminantes emergentes presentes en estas aguas y constituyen una base para diseñar futuras investigaciones sobre los problemas ecotoxicológicos que pueden generar estos compuestos en la zona donde se usan estas aguas residuales.

\section{Remoción de compuestos en la PTAR}

De manera individual, los compuestos más persistentes en los efluentes de la PTAR-Atapaneo 
CUADRO III. CUANTIFICACIÓN DE CONTAMINANTES EMERGENTES DETECTADOS POR ESPECTROMETRÍA DE MASAS CON IONIZACIÓN POR ELECTRONEBULIZACIÓN Y DETECTOR DE TIEMPO DE VUELO (ESI-MS-TOF)

\begin{tabular}{|c|c|c|c|c|c|}
\hline \multirow{2}{*}{ Compuesto } & \multicolumn{2}{|l|}{ Influente $\mu \mathrm{g} / \mathrm{L}$} & \multicolumn{2}{|l|}{ Efluente $\mu \mathrm{g} / \mathrm{L}$} & \multirow{2}{*}{$\begin{array}{l}\text { Valores de referencia para } \\
\text { influentes } \mu \mathrm{g} / \mathrm{L}\end{array}$} \\
\hline & Rango & Promedio & Rango & Promedio & \\
\hline Tetraciclina & $2.2-160.2$ & 72.7 & $2.7-119.4$ & 64.9 & $0.98-700$ (Man-Hong et al. 2015) \\
\hline Cefaclor & $0.8-15.3$ & 3.2 & $1.1-12.1$ & 2.3 & 0.108 (Rivera et al. 2013) \\
\hline Cefadroxilo & $3.0-18.1$ & 5.0 & $1.3-15.0$ & 4.7 & $0.23-1.05$ (Won et al. 2011) \\
\hline Ampicilina & $1.9-44.0$ & 17.1 & $2.0-37.7$ & 15.5 & 0.017 (Michael et al. 2013) \\
\hline Clonazepam & $0.2-2.8$ & 1.3 & $0.1-1.3$ & 0.7 & 0.0437 (Loos et al. 2013) \\
\hline Lormetazepam & $1.5-30.7$ & 13.5 & $2.2-27.4$ & 12.2 & $0.02-0.1$ (Racamonde et al. 2014) \\
\hline Secobarbital & $0.4-7.4$ & 2.1 & $0.4-5.1$ & 1.0 & 5-30 (Daughton 2014) \\
\hline Maprotilina & $0.8-15.7$ & 4.3 & $0.6-14.6$ & 4.2 & 0.0165 (Loos et al. 2013) \\
\hline Levotiroxina & $3.1-55.0$ & 6.1 & $7.3-37.6$ & 5.4 & No reportado \\
\hline Cis-androsterona & $0.7-9.5$ & 2.9 & $1.2-7.3$ & 2.6 & 5.604 (Bizkarguenaga et al. 2012) \\
\hline Paracetamol & $0.1-4.3$ & 0.6 & $0.1-1.0$ & 0.2 & 2.1 (Rivera et al. 2013) \\
\hline Lidocaína & $0.4-11.0$ & 1.9 & $0.1-7.7$ & 1.4 & $0.023-0.743$ (Rúa y Püttmann 2013) \\
\hline Bromfeniramina & $2.6-19.2$ & 5.5 & $2.4-18.6$ & 5.4 & 0.04 (Bisceglia et al. 2010) \\
\hline Fexofenadina & $0.6-147.9$ & 31.1 & $1.2-33.6$ & 12.7 & 1.287 (Loos et al. 2013) \\
\hline Amfetamina & $0.2-1.3$ & 0.5 & $0.1-0.8$ & 0.1 & 0.16 (Mendoza et al. 2014) \\
\hline Morfina & $1.0-14.1$ & 3.5 & $1.0-12.0$ & 3.2 & $0.021-0.148$ (Mendoza et al. 2014) \\
\hline Benzoilecgonina & $2.1-18.1$ & 4.5 & $1.5-4.8$ & 2.4 & 0.520 (van Nuijs et al. 2009) \\
\hline 11-nor- $\Delta 9$-THC-9-COOH & $0.7-46.0$ & 12.1 & $0.7-36.0$ & 8.4 & $0.04-0.07$ (Mendoza et al. 2014) \\
\hline Dimetilamfetamina & $0.6-4.0$ & 1.6 & $0.1-3.3$ & 1.2 & 0.31 (Mendoza et al. 2014) \\
\hline Fenciclidina & $0.6-9.8$ & 1.7 & $0.9-7.0$ & 1.1 & No reportado \\
\hline Metadona & $0.1-1.8$ & 0.1 & $0.0-1.2$ & 0.1 & $0.007-0.054$ (Mendoza et al. 2014) \\
\hline Polietilenglicol & $67.8-588.2$ & 135.3 & $64.6-564.2$ & 136.0 & No reportado \\
\hline
\end{tabular}

fueron los siguientes: polietilenglicol, maprotilina, bromfeniramina, cefadroxilo, metadona, lormetazepam, cis-androsterona, ampicilina y morfina, con eficiencias de remoción de $0,1.2,2.4,5.8,7.7,9.5,9.6$ y $9.7 \%$, respectivamente. Por grupos, los compuestos hormonales y esteroides mostraron mayor persistencia en los efluentes, con una eficiencia media de remoción de $10.7 \%$, seguidos de antibióticos (13.6\%), ansiolíticos (26.6\%), anestésicos (27.7\%), antihistamínicos (30.8\%), drogas de abuso (33.3\%) y analgésicos $(63.9 \%)$. Considerando las concentraciones medias de los contaminantes emergentes en influentes y efluentes, se observa que el sistema de tratamiento de la PTAR-Atapaneo (lodos activados) tiene una eficiencia de remoción media de $25.8 \%$, variando con el tipo de compuesto y con extremos de 0 a $74.5 \%$ para el polietilenglicol y la amfetamina, respectivamente. De acuerdo con las concentraciones medias de los compuestos detectados en los efluentes y una capacidad de tratamiento de la PTAR-Atapaneo de $1200 \mathrm{~L} / \mathrm{s}$, se calcula, de forma gruesa, que se agregan anualmente unos $9000 \mathrm{~g}$ de estos contaminantes en las aguas tratadas. Otros autores (Xu et al. 2014) reportan descargas de $4800 \mathrm{~g} / \mathrm{año}$ de contaminantes emergentes (disruptores endócrinos) en las aguas residuales tratadas.

\section{Efectos potenciales en el ambiente de los conta- minantes emergentes analizados}

Los efluentes de la PTAR-Atapaneo son vertidos al cauce del río Grande de Morelia donde se mezclan con otros flujos de AR urbano industriales no tratadas y son conducidos finalmente al lago de Cuitzeo, área con importantes actividades turísticas y de pesca; una parte de esta agua es utilizada en el riego de cultivos en la zona Morelia-Queréndaro. La descarga de los contaminantes emergentes detectados hacia estos ambientes, por tanto, puede estar causando severos problemas toxicológicos. Diversos autores han encontrado que los productos farmacéuticos en el ambiente pueden afectar a los organismos acuáticos causando toxicidad, genotoxicidad, alteración de procesos endócrinos y desarrollo de resistencia a patógenos (Rosal et al. 2010, Ortíz de García et al. 2014). Los antibióticos pueden inducir resistencia en los microorganismos del suelo (Negreanu et al. 2012, Chaoqi et al. 2014) y esta resistencia puede ser transmitida a microorganismos que representan un riesgo potencial para la salud humana (Novo et al. 2013). Estudios específicos sobre la tetraciclina indican que es un compuesto de alta persistencia y puede acumularse en los suelos y plantas cultivadas como el arroz (Boonsaner y Hawker 2012). Las 
benzodiacepinas, donde se incluye el clonazepam y el lormetazepam, tienen efectos negativos sobre el comportamiento de peces, disminuyendo su audacia, actividad y socialización (Brodin et al. 2013). Este grupo de compuestos es poco susceptible a la degradación debido a la presencia de elementos halógenos en su estructura química, de ahí que los tratamientos biológicos en aguas residuales sean poco efectivos ( $<10 \%$ de eficiencia). Los lodos activados pueden reducir su concentración en un 10 a $50 \%$ (Kosjek et al. 2012). No se tienen referencias específicas de los efectos ambientales de la cis-androsterona y la levotiroxina, pero se han encontrado evidencias de otros productos hormonales que aún en bajas concentraciones $(5.2 \mathrm{ng} / \mathrm{L})$ pueden inhibir las características sexuales secundarias en machos de peces y causar feminización (Xu et al. 2014). Estos compuestos se acumulan en los tejidos de peces y pueden pasar a los seres humanos donde aumentan la posibilidad de generar cáncer de mama y testículo (Shan et al. 2011).

En relación con la benzoilecgonina, el principal metabolito de la cocaína, se ha encontrado que concentraciones de $1 \mathrm{ng} / \mathrm{L}$ pueden causar alteraciones en la actividad mitocondrial y en los niveles de ADN en esporas de helechos afectando la germinación de los gametofitos (García-Campero et al. 2015). El paracetamol, por otro lado, es un compuesto tóxico y persistente ante los procesos de tratamiento de las PTAR (Rivera et al. 2013). En organismos acuáticos como la anguila (Anguilla anguilla) inhibe la actividad de la acetilcolinesterasa causando neurotoxicidad (Nunes et al. 2015) y en almejas ocasiona estrés oxidativo que deriva en necrosis y muerte celular (Correia et al. 2016). No se encontraron referencias sobre los efectos en la biota acuática de otros compuestos detectados en este trabajo -maleato de bromfeniramina, fexofenadina, lidocaína, fenciclidina y polietilenglicol-, pero pueden ser la base para futuras investigaciones ecotoxicológicas. Es necesario, por tanto, diseñar investigaciones sobre la dinámica, transporte y efectos ambientales y sanitarios de los contaminantes emergentes que se están virtiendo en la zona a través de las aguas residuales crudas y tratadas.

Por otro lado, la presencia de contaminantes emergentes en los efluentes de la PTAR-Atapaneo, evidencia la necesidad de buscar métodos alternativos de tratamiento de las AR que sean más eficientes en la remoción o eliminación de estos compuestos y así disminuir el riesgo de causar efectos tóxicos a la biota acuática y a microorganismos del suelo en aquellos terrenos donde estas aguas son utilizadas para riego agrícola. Los procesos fotocatalíticos heterógeneos, la ozonificación y la electrocoagulación son algunos de los métodos que actualmente se están investigando para la degradación de compuestos orgánicos y contaminantes emergentes (Rosal et al. 2010, PérezSicairos et al. 2011).

\section{CONCLUSIONES}

El presente trabajo confirma, mediante un análisis integral, la calidad de los influentes y efluentes de la PTAR-Atapaneo, Morelia. El acoplamiento de los análisis físicos y químicos, absorción atómica, espectroscópicos (FT-IR) y espectrométricos (ESIMS-TOF) en el estudio de las AR permite mostrar un panorama más claro sobre el reúso potencial de estos flujos en el riego agrícola. Los resultados sobre la hidroquímica de las AR muestran que el sistema de tratamiento reduce el contenido de sales y metales tóxicos. En relación con los contaminantes emergentes detectados (antibióticos, ansiolíticos, hormonas, analgésicos, anestésicos, antihistamínicos, drogas de abuso, polietilenglicol) es necesario realizar investigaciones sobre su dinámica, transporte y efectos sobre los ecosistemas debido a las altas concentraciones encontradas en los efluentes; los trabajos posteriores, sin embargo, deberán contemplar separaciones cromatográficas y análisis por metodologías recomendadas como LC-ESI-TOF o LC/MS/MS. Dado que el sistema de tratamiento de AR usado en la PTARAtapaneo es poco eficiente para la eliminación de los contaminantes emergentes, se requiere implementar métodos alternativos más efectivos y que puedan ser adaptados en las PTAR. Actualmente se cuenta con alternativas como la electrocoagulación, el intercambio iónico, la precipitación y oxidación químicas, la adsorción, la ultrafiltración, la ósmosis inversa, la electrodiálisis y la ozonificación. Los resultados obtenidos constituyen una base para desarrollar propuestas de reúso de aguas residuales tratadas y de regulación sanitaria, especialmente en lo relativo al establecimiento de límites máximos permitidos para contaminantes emergentes (antibióticos, analgésicos, antihistamínicos, drogas y otros compuestos) en efluentes empleados en el riego agrícola.

\section{AGRADECIMIENTOS}

Los autores agradecen el apoyo del Instituto Politécnico Nacional (Centro Interdisciplinario de Investigación para el Desarrollo Integral Regional, Unidad Michoacán y del Centro de Nanociencias y 
Micro y Nanotecnologías, del Colegio de Postgraduados y de la Universidad Michoacana de San Nicolás de Hidalgo (Facultad de Químico Farmacobiología) para la realización de este trabajo.

\section{REFERENCIAS}

Anderson R.J., Bendel D.J. y Groundwater P.W. (2004). Organic spectroscopic analysis. 5. Mass spectrometry. Royal Society of Chemistry, Cambridge, Reino Unido, 176 pp. DOI: 10.1039/9781847551566

Ayers R. S. y Westcot D. (1994). Water quality for agriculture. FAO Irrigation and Drainage Paper. 29 Rev. 1. Reprinted. Food and Agriculture Organization of the United Nations, Rome, Italy, $174 \mathrm{pp}$.

Bisceglia K. J., Yu J.T., Coelhan M., Bouwer E.J. y Roberts A.L. (2010). Trace determination of pharmaceuticals and other wastewater-derived micropollutants by solid phase extraction and gas chromatography/mass spectrometry. J. Chromatogr. A. 1217 (4), 558-564. DOI: 10.1016/j.chroma.2009.11.062

Bizkarguenaga E., Ros O., Iparraguirre A., Navarro P., Vallejo A., Usobiaga A. y Zuloaga O. (2012). Solid-phase extraction combined with large volume injection-programmable temperature vaporization-gas chromatography-mass spectrometry for the multiresidue determination of priority and emerging organic pollutants in wastewater. J. Chromatogr. A. 1247, 104-117. DOI: 10.1016/j.chroma.2012.05.022

Boonsaner M. y Hawker D.W. (2012). Investigation of the mechanism of uptake and accumulation of zwitterionic tetracyclines by rice (Oryza sativa L.). Ecotox. Environ. Safe. 78, 142-147.

DOI: 10.1016/j.ecoenv.2011.11.023

Brodin T., Fick J., Jonsson M. y Klaminder J. (2013). Dilute concentrations of a psychiatric drug alter behavior of fish from natural populations. Science 339 (6121), 814-815. DOI: $10.1126 /$ science. 1226850

Chaoqi C., Jing L., Peipei Ch., Ru D., Pengfei Z. y Xiging L. (2014). Occurrence of antibiotics and antibiotic resistances in soils from wastewater irrigation areas in Beijing and Tianjin, China. Environ. Pollut. 193, 94101. DOI: 10.1016/j.envpol.2014.06.005

Cherfi A., Achour M., Cherfic M., Otmani S. y Morsli A. (2015). Health risk assessment of heavy metals through consumption of vegetables irrigated with reclaimed urban wastewater in Algeria. Process Saf. Environ. Protec. 98, 245-252. DOI: 10.1016/j.psep.2015.08.004 CONAGUA (2015). Atlas del agua en México 2014. Comisión Nacional del Agua [en línea]. http://www. conagua.gob.mx/CONAGUA07/Publicaciones/Publicaciones/SGP-17-14.pdf 15/03/2015.
Correia B., Freitas R., Figueira E., Soares A.M.V.M. y Nunes B. (2016). Oxidative effects of the pharmaceutical drug paracetamol on the edible clam Ruditapes philippinarum under different salinities. Comp. Biochem. Physiol. C. 179, 116-124.

DOI: $10.1016 /$ j.cbpc.2015.09.006

Daughton C.G. (2014). Eco-directed sustainable prescribing: feasibility for reducing water contamination by drugs. Sci. Total Environ. 493, 392-404. DOI: 10.1016/j.scitotenv.2014.06.013

Durán-Álvarez J.C., Prado B., González D., Sánchez Y. y Jiménez-Cisneros B. (2014). The transport of three emerging pollutants through an agricultural soil irrigated with untreated wastewater. J. Water Reuse Desal. 4 (1), 9-17. DOI: 10.2166/wrd.2013.003

Durán-Álvarez J.C., Prado B., González D., Sánchez Y. y Jiménez-Cisneros B. (2015). Environmental fate of naproxen, carbamazepine and triclosan in wastewater, surface water and wastewater irrigated soil - Results of laboratory scale experiments. Sci. Total Environ. 538, 350-362. DOI: 10.1016/j.scitotenv.2015.08.028

Eaton A. D., Clesceri L. S., Rice E. W. y Greesberg A. E. (2005). Standard methods for the examination of water and wastewater. 21 Ed., American Public Health Association, Washington, EUA, 1208 pp.

García-Cambero J.P, García-Cortés H., Valcárcel Y. y Catalá M. (2015). Environmental concentrations of the cocaine metabolite benzoylecgonine induced sublethal toxicity in the development of plants but not in a zebrafish embryo-larval model. J. Hazard Mater. 300, 866-872. DOI: 10.1016/j.jhazmat.2015.08.019

Idowu O.J, Lombard K.A., Hyder D. y Ulery A.L. (2015). Soil quality of a semi-arid pasture irrigated with reverse osmosis wastewater-A case study from Northern New Mexico. JWARP. 7, 1121-1130.

DOI: $10.4236 /$ jwarp.2015.714092

Jee R.D. (2011). Infrared spectroscopy. En: Clarke's analysis of drugs and poisons. (A. C. Moffat, M.D. Osselton y D. Widdop, Ed.). 4 ed. Pharmaceutical Press, Londres, Reino Unido, pp. 521-538.

Karvelas M., Katsoyiannis A. y Samara C. (2003). Occurrence and fate of heavy metals in the wastewater treatment process. Chemosphere 53 (10), 1201-1210. DOI: $10.1016 / \mathrm{S} 0045-6535(03) 00591-5$

Kim M., Han D. y Kim D. (2015). Selective release of phosphorus and nitrogen from waste activated sludge with combined thermal and alkali treatment. Bioresource Technol. 190, 522-528. DOI: 10.1016/j.biortech.2015.01.106

Kornboonraksa T. (2016). Using tertiary-treated municipal wastewater as makeup water by reverse osmosis membrane. Desal. Water Treat. 57 (16), 7422-7431. DOI: $10.1080 / 19443994.2015 .1024937$ 
Kosjek T., Perko S., Zupanc M., Zanoski H. M., Landeka D. T., Zigon D., Kompare B. y Heath E. (2012). Environmental occurrence, fate and transformation of benzodiazepines in water treatment. Water Res. 46 (2), 355-368. DOI: 10.1016/j.watres.2011.10.056

Kümmerer K. (2011). Emerging contaminants. Treatise on Water Science 3, 69-87.

DOI: 10.1016/B978-0-444-53199-5.00052-X

Loos R., Carvalho R., Antonio D.C., Comero S., Locoro G., Tavazzi S., Paracchini B., Ghiani M., Lettieri T., Blaha L., Jarosova B., Voorspoels S., Servaes K., Haglund P., Fick J., Lindberg R. H., Schwesig D. y Gawlik B. M. (2013). EU-wide monitoring survey on emerging polar organic contaminants in wastewater treatment plant effluents. Water Res. 47 (17), 64756487. DOI: $10.1016 /$ j.watres.2013.08.024

Man-Hong H., Wei Z., Chong L. y Hong-Ying H. (2015). Fate of trace tetracycline with resistant bacteria and resistance genes in an improved AAO wastewater treatment plant. Proc. Saf. Environ. Prot. 93, 68-74. DOI: 10.1016/j.psep.2014.04.004

Mendoza A., López de Alda M., González S., Mastroianni N., Barceló D. y Valcárcel Y. (2014). Occurrence of drugs of abuse and benzodiazepines in river waters from the Madrid Region (Central Spain). Chemosphere 95, 247-255.

DOI: 10.1016/j.chemosphere.2013.08.085

Michael I., Rizzo L., McArdell C.S., Manaia C.M., Merlin C., Schwartz T., Dagot C. y Fatta-Kassinos D. (2013). Urban wastewater treatment plants as hotspots for the release of antibiotics in the environment: A review. Water Res. 47 (3), 957-995.

DOI: $10.1016 /$ j.watres.2012.11.027

Moffat A. C., Osselton M.D y Widdop D. (2011). Clarke's analysis of drugs and poisons. 4 Ed. Pharmaceutical Press, Londres, Reino Unido, 2609 pp.

Negreanu Y., Pasternak Z., Jurkevitch E. y Cytryn E. (2012). Impact of treated wastewater irrigation on antibiotic resistance in agricultural soils. Environ. Sci. Technol. 46 (9), 4800-4808. DOI: 10.1021/es204665b

Novo A., André S., Viana P., Nunes O. C. y Manaia C. M. (2013). Antibiotic resistance, antimicrobial residues and bacterial community composition in urban wastewater. Water Res. 47 (5), 1875-1887.

DOI: 10.1016/j.watres.2013.01.010

Nunes B., Verde M.F. y Soares A.M.V.M. (2015). Biochemical effects of the pharmaceutical drug paracetamol on Anguilla anguilla. Environ. Science Pollut. Res. 22 (15), 11574-11584.

DOI: $10.1007 / \mathrm{s} 11356-015-4329-6$

Ortiz de García S.A., Pinto-Pinto G., García-Encina P.A. y Irusta-Mata R. (2014). Ecotoxicity and environmental risk assessment of pharmaceuticals and personal care products in aquatic environments and wastewater treatment plants. Ecotoxicology 23 (8), 1517-1533. DOI: $10.1007 / \mathrm{s} 10646-014-1293-8$

Pérez-Sicairos S., Morales-Cuevas J.B., Félix-Navarro R.M. y Hernández-Calderón O.M. (2011). Evaluation of the electro-coagulation process for the removal of turbidity of river water, wastewater and pond water. Rev. Mex. Ing. Quim. 10, 79-91.

PROFECO (2010). Sondeo en línea sobre hábitos de consumo de medicamentos. Procuraduría Federal del Consumidor [en línea]. http://www.profeco.gob. $\mathrm{mx} / \mathrm{encuesta} / \mathrm{mirador} / 2010 / \mathrm{cons} \_$med_2010.pdf 10/09/2015.

Racamonde I., Rodil R., Quintana J.B., Villaverde-de-Sáa E. y Cela R. (2014). Determination of benzodiazepines, related pharmaceuticals and metabolites in water by solid-phase extraction and liquid-chromatographytandem mass spectrometry. J. Chromatogr. A. 1352, 69-79. DOI: 10.1016/j.chroma.2014.05.064

Rivera J., Sánchez M., Ferro M. A., Prados G. y Ocampo R. (2013). Pharmaceuticals as emerging contaminants and their removal from water. A review. Chemosphere 93 (7), 1268-1287.

DOI: 10.1016/j.chemosphere.2013.07.059

Robles-Molina J., Gilbert-López B., García-Reyes J.F. y Molina-Díaz A. (2010). Determination of organic priority pollutants in sewage treatment plant effluents by gas chromatography high-resolution mass spectrometry. Talanta 82 (4), 1318-1324. DOI: 10.1016/j.talanta.2010.06.053

Rocha-Gutiérrez B.A., Peralta-Pérez M. R. y Zavala-Díaz F.J. (2015). Revisión global de los contaminantes emergentes PBDE y el caso particular de México. Rev. Int. Contam. Ambie. 31, 311-320.

Rodier J., Legube B. y Merlet N. (2011). Análisis del agua. 9 Ed. Editorial Omega, Barcelona, España, 1477 pp.

Rosal R., Rodríguez A., Perdigón-Melón J.A., Petre A., García-Calvo E., Gómez M.J., Agüera A. y FernándezAlba A.R. (2010). Occurrence of emerging pollutants in urban wastewater and their removal through biological treatment followed by ozonation. Water Res. 44 (2), 578-588. DOI: 10.1016/j.watres.2009.07.004

Rúa P. C. y Püttmann W. (2013). Degradation of lidocaine, tramadol, venlafaxine and the metabolites O-desmethyltramadol and O-desmethylvenlafaxine in surface waters. Chemosphere 90 (6), 1952-1959.

DOI: 10.1016/j.chemosphere.2012.10.039

SEMARNAT (1997). Norma Oficial Mexicana NOM001-ECOL-1996. Límites máximos permisibles de contaminantes en las descargas de aguas residuales en aguas y bienes nacionales. Secretaría de Medio Ambiente, Recursos Naturales y Pesca. Diario Oficial de la Federación. 6 de enero de 1997. 
Shan L., Guang-Guo Y., Jian-Liang Z., Feng Ch., Bin Y., Li-Jun Z. y Hua-Jie L. (2011). Trace analysis of 28 steroids in surface water, wastewater and sludge samples by rapid resolution liquid chromatographyelectrospray ionization tandem mass spectrometry. J. Chromatogr. A. 1218 (10), 1367-1378.

DOI: $10.1016 /$ j.chroma.2011.01.014

Silverstein R.M., Webster F.X. y Kiemle D.J. (2005). Spectrometric identification of organic compounds. 1. Mass spectrometry. John Wiley and Sons, Inc. Madison, EUA, 502 pp.

Stimpfl T. (2011). Extraction. En: Clarke's analysis of drugs and poisons. (A. C. Moffat, M.D. Osselton y D. Widdop, Eds.). 4 Ed. Pharmaceutical Press, Londres, Reino Unido, pp. 458-470.

Tadeo J. L., Sánchez C., Albero B., García A. I. y Pérez R. A. (2012). Analysis of emerging organic contaminants in environmental solid samples. Centr. Eur. J. Chem. 10 (3), 480-520. DOI: 10.2478/s11532-011-0157-9

van Nuijs A. L. N., Pecceub B., Theunisc L., Duboisc N., Charlierc C., Jorensd P.G., Bervoetsb L., Blustb R., Neelsa H. y Covaci A. (2009). Spatial and temporal variations in the occurrence of cocaine and benzoylecgonine in waste- and surface water from Belgium and removal during wastewater treatment. Water Res. 43 (5), 1341-1349. DOI: 10.1016/j.watres.2008.12.020
Verlicchi P., Al Aukidya M. y Zambello E. (2012). Occurrence of pharmaceutical compounds in urban wastewater: Removal, mass load and environmental risk after a secondary treatment-A review. Sci. Total Environ. 429, 123-155. DOI: 10.1016/j.scitotenv.2012.04.028

Verlicchi P. y Zambello E. (2015). Pharmaceuticals and personal care products in untreated and treated sewage sludge: Occurrence and environmental risk in the case of application on soil - A critical review. Sci. Total Environ. 538, 750-767.

DOI: $10.1016 /$ j.scitotenv.2015.08.108

Won J. S., Ji-Woo L., Eung-Sun L., Sun-Kyoung S., Seung R. y Jeong-Eun O. (2011). Occurrence and distribution of pharmaceuticals in wastewater from households, livestock farms, hospitals and pharmaceutical manufactures. Chemosphere 82 (2), 179-186.

DOI: $10.1016 /$ j.chemosphere.2010.10.026

Xu E.G.B., Liu S., Ying G., Zheng G.J.S., Lee J.H.W. y Leung K.M.Y. (2014). The occurrence and ecological risks of endocrine disrupting chemicals in sewage effluents from three different sewage treatment plants and in natural seawater from a marine reserve of Hong Kong. Mar. Pollut. Bull. 85 (2), 352-362. DOI: 10.1016/j.marpolbul.2014.02.029 